\title{
Characterization of a novel toxin-antitoxin module, VapBC, encoded by Leptospira interrogans chromosome
}

\author{
Yi Xuan ZHANG ${ }^{1,2}$, Xiao Kui GUO ${ }^{3}$, Chuan $\mathrm{WU}^{2}$, Bo BI ${ }^{1}$, Shuang Xi REN ${ }^{4}$, Chun Fu WU ${ }^{1}$, Guo Ping ZHAO ${ }^{2,} 4^{*}$ \\ ${ }^{1}$ Pharmaceutical Department, Shenyang Pharmaceutical University, 103 Wenhua Road, Shenhe District, Shenyang 110016,China. \\ ${ }^{2}$ Research Center of Biotechnology, Shanghai Institutes for Biological Sciences, Chinese Academy of Sciences, 500 Caobao \\ Road, Shanghai 200233, China. \\ ${ }^{3}$ Department of Microbiology and Parasitology, Shanghai Second Medical University, 280 Chongqingnan Road, Shanghai \\ 200025, China. \\ ${ }^{4}$ Chinese National Human Genome Center at Shanghai (CHGCS), 250 Bibo Road, ZhangJiang High Tech Park, Shanghai, 201203, \\ China.
}

\begin{abstract}
Comparative genomic analysis of the coding sequences (CDSs) of Leptospira interrogans revealed a pair of closely linked genes homologous to the $v a p B C$ loci of many other bacteria with respect to both deduced amino acid sequences and operon organizations. Expression of single vapC gene in Escherichia coli resulted in inhibition of bacterial growth, whereas co-expression of $\operatorname{vap} B C$ restored the growth effectively. This phenotype is typical for three other characterized toxin-antitoxin systems of bacteria, i.e., mazEF[1], relBE[2] and chpIK[3]. The VapC proteins of bacteria and a thermophilic archeae, Solfolobus tokodaii, form a structurally distinguished group of toxin different from the other known toxins of bacteria. Phylogenetic analysis of both toxins and antitoxins of all categories indicated that although toxins were evolved from divergent sources and may or may not follow their speciation paths (as indicated by their 16s RNA sequences), co-evolution with their antitoxins was obvious.
\end{abstract}

Keywords: Leptospria, toxin-antitoxin system, VapBC, co-evolution, speciation.

\section{INTRODUCTION}

Leptospira interrogans is the etiologic agent of leptospirosis, which is a worldwide zoonosis with a much greater incidence in tropical and subtropical regions[4]. The recently reported genomic sequence of the $L$. interrogans serogroup Icterohaemorrhagiae serovar lai [5] indicated that in contrast to the two strictly parasitic spirochetes, Treponema pallidum [6] and Borrelia burg-dorferi [7], L. interrogans possessed much more physiologically important genes for its facultative free-living metabolism, allowing it to adapt to its diverse environment. However, the regulation of the physiology is largely unknown.

Toxin-antitoxin (TA) modules are of importance among bacterial regulatory systems[8]. They were firstly found on plasmids and considered prevalently to be associated with stable plasmid inheritance at cell division[9-11]. The well-known plasmid-encoded TA loci contained CcdA/ CcdB of F factors[12, 13], Kis/Kid of R1[14], Phd/Doc of P1[15], ParD/ParE of RK2[16], pas locus of pTF-CF2

"Correspondence: Guo Ping ZHAO, Tel: +86-21-50801919; Fax: +86-21-50801922; E-mail: gpzhao@sibs.ac.cn.
[17], $\omega-\varepsilon-\xi$ operon of pSM19035[18], stb locus of pMYSH6000[19] and relBE locus of P307[20].

Recently, TA modules were also identified on chromosomes and considered to be associated with host stress response[21]. Only three TA loci families encoded by chromosomes have been identified so far, relBE[2], mazEF [1], and chpIK[3]. Generally, TA loci are organized into operons where the first cistron encodes a small instable antitoxin while the second cistron encodes a large stable antitoxin[21].

The presence of a TA module in L. interrogans was firstly reported by Picardeau et al[3] by characterizing the large chromosome (CI) encoded chp locus (chpI-chpK). It was proposed that the presence of this system might provide means for bacterial adaptation to a poor nutrition environment. Further analysis of the L. interrogans genome found a $m a z E F$ locus (LA1780/LA1781) belonging to the TA system. When a pair of $\operatorname{vap} B C$ (virulence-associated protein) genes were cloned and expressed in Escherichia coli for functional analysis, we noticed that the characteristics of these genes and their products were similar to that of the TA systems, but unlikely to be associated 
Tab 1. Leptospira strains used in this study

\begin{tabular}{|c|c|c|c|}
\hline Strains & Series code* & Source & Virulence \\
\hline \multicolumn{4}{|l|}{ L. interrogans strains: } \\
\hline serogroup Icterheamorrhagiae serovar lai & 56601 & $\mathrm{CCDC}$ & + \\
\hline serogroup Ballum serovar ballum & 56604 & CCDC & + \\
\hline serogroup Pyrogenes serovar pyrogenes & 56605 & $\mathrm{CCDC}$ & + \\
\hline serogroup Autumnalis serovar autumnalis & 56606 & $\mathrm{CCDC}$ & + \\
\hline serogroup Pomona serovar pomona & 56608 & $\mathrm{CCDC}$ & + \\
\hline serogroup Grripotypphosa serovar lin 6 & 56609 & $\mathrm{CCDC}$ & + \\
\hline serogroup Hebdomadis serovar hebdomadis & 56610 & $\mathrm{CCDC}$ & + \\
\hline serogroup Icterheamorrhagiae serovar lai & - & Inst. Pasteur & - \\
\hline \multicolumn{4}{|l|}{ L. biflexa strains: } \\
\hline serogroup Semaranga serovar monvalerio & 57001 & $\mathrm{CCDC}$ & - \\
\hline serogroup Semaranga serovar patoc & 651505 & $\mathrm{CCDC}$ & - \\
\hline
\end{tabular}

*Code numbers of Chinese Center for Disease Control and Prevention

Tab 2. Oligonucleotide primers specific for $v a p B$ and vapC used in the PCR and RT-PCR reactions

\begin{tabular}{|c|c|c|c|c|}
\hline Genes & Reactions & Direction & Primer sequence* & Length (bp) \\
\hline \multirow[t]{2}{*}{$v a p B$} & PCR & Sense & 5'CGGAGATAACATATGCAAACAGCCAAATT 3' & 351 \\
\hline & & Antisense & 5'ACTCAGGATCCGCAACAGTTAAAGACGAAACAAA3' & \\
\hline \multirow[t]{2}{*}{$\operatorname{vap} C$} & PCR & Sense & 5’AAAAGCTCATATGTATCTTTTGGATA3' & 443 \\
\hline & & Antisense & 5'CGCGGATCCGAAGTTAGTTGTAGTGG3' & \\
\hline \multirow[t]{2}{*}{$\operatorname{vap} B C$} & PCR & Sense & 5'CGGAGATAACATATGCAAACAGCCAAATT3' & 674 \\
\hline & & Antisense & 5'CGCGGATCCGAAGTTAGTTGTAGTGG3' & \\
\hline \multirow[t]{2}{*}{$\operatorname{vap} B C$} & RT-PCR & Sense & 5’AGTCAAGCGGTTCGACTACCA 3' & 487 \\
\hline & & Antisense & 5'GTCTATTGAACCGATTACATTACCT 3' & \\
\hline
\end{tabular}

*The designed restriction sites incorporated into the primers, NdeI for the sense sequences and BamHI for the antisense primers are bolded and underlined.

with virulence. Based on their sequence homology and operon organization, a novel group of TA system was characterized.

\section{MATERIALS AND METHODS}

\section{Materials}

Plasmid pET28b (+) from Novagen (Madison, Wisconsin, USA) was used for heterogeneous gene expression. Golden Taq DNA polymerase, BigDye Terminator Cycle Sequencing Ready Reaction Kit, restriction enzymes, T4 DNA ligase, and bacterial alkaline phosphatase were obtained from Takara (China). Trizol reagent and Freund's complete and incomplete adjuvant was obtained from GBICO-BRL (USA). Vector plasmid pUCm-T, AMV Reverse Transcription System, goat anti-rabbit immuno-globulin G (Fc)-Alkaline Phosphatase conjugated and BCIP/NBT color development substrate were purchased from Promega (USA). Nitrocellulose membranes were obtained from Amersham (USA). All other reagents were of the highest purity available.

\section{Bacterial strains and culture conditions}

Leptospira strains used in this work are shown in Tab 1. The avirulent $L$. interrogans strain of serovar lai was kindly supplied by Prof. Saint Girons Unité de Bactériologie Moléculaire et Médicale, Institut Pasteur, France. All of the other strains were maintained by the Institute for Infectious Disease Control and Prevention (IIDC), Chinese Center for Disease Control and Prevention (CCDC), Beijing, China. The strains were cultured in EMJH(Johnson and Marris modification of the Ellinghausen and McCullough medium[4]) at $28^{\circ} \mathrm{C}$ for 3-4 $\mathrm{d}$ under aerobic conditions until mid-log phase was reached (about $10^{8}$ bacteria $/ \mathrm{ml}$ ). Bacterial counts were determined by dark-field microscopy. E. coli strains DH5 $\alpha$ and BL21(DE3/pLysS) used as hosts for cloning or expression were cultured in LB (LuriaBertani) medium.

\section{Databases and softwares}

The complete genomic sequence of the virulent $L$. interrogans serogroup Icterohaemorrhagiae serovar lai was obtained from http:// www.chgc.sh.cn/lep/. BLAST searching the NCBI non-redundant 
Tab 3. Vectors used in this study and plasmids with cloned genes

\begin{tabular}{lll}
\hline Plasmids & \multicolumn{1}{c}{ Construction } & \multicolumn{1}{c}{ Source } \\
\hline pUB & ApUC19 derivative carrying the vapB gene & This work \\
pUC & ApUC19 derivative carrying the vapC gen & This work \\
pUBC & A pUC19 derivative carrying the vapBC gene & This work \\
pET28b $(+)$ & Expression vector with strong T7 promotor, kan & Novagen (USA) \\
pTB & A pET28b derivative carrying the vapB gene, kan $^{\mathrm{r}}$ & This work \\
pTC & A pET28b derivative carrying the $\operatorname{vapC}$ gene, $\operatorname{kan}^{\mathrm{r}}$ & This work \\
pTBC & A pET28b derivative carrying the $\operatorname{vapBC}$ gene, $\mathrm{kan}^{\mathrm{r}}$ & This work \\
\hline
\end{tabular}

nucleotide database, or non-redundant protein database on SwissProt/ TrEMBL were used to annotate the $L$. interrogans vap $B C$ and $m a z E F$ genes. Bacterial protein sequences of $m a z E F$, vapBC, chpIK and relBE were obtained from the genome database of KEGG by employing the TFASTA search (http://fasta.genome.ad.jp/ideas/fasta/ tfasta_genome.html) with inputting VapB, VapC, MazE, MazF, ChpI and $\mathrm{ChpK}$ protein sequences of $L$. interrogans. Only those with similarity scores above e- 8 were selected for further analysis. Multiple-sequence alignment was accomplished by using Bioedit. Phylogenetic trees were constructed using Mega 2.0.

\section{Expressing vapB and vapC genes in $E$. coli and protein purification}

PCR primers for L. interrogans vapB, vapC and vapBC genes were designed as shown in Tab 2. Forward primers incorporated an $\mathrm{NdeI}$ restriction site and reverse primers incorporated a $\mathrm{BamHI}$ restriction site. The reverse primers were designed to be immediately behind the terminator "TTA" to ensure involvement of the terminator in the PCR product. All the genes were amplified using Leptospira spp. genomic DNA as templates, which were extracted from mid-log phase cultures of corresponding strains grown in EMJH medium[22]. PCR was performed using the following cycle; $95^{\circ} \mathrm{C}$ for $10 \mathrm{~min}$, followed by $30 \mathrm{cycles}$ at $94^{\circ} \mathrm{C}$ for $30 \mathrm{sec}$ for denaturing, $54^{\circ} \mathrm{C}$ for $30 \mathrm{sec}$ for $v a p B$ or $58^{\circ} \mathrm{C}$ for $1 \mathrm{~min}$ for vapC and vapBC for annealing, and $72^{\circ} \mathrm{C}$ for $1 \mathrm{~min}$ for reaction. The whole reaction was completed at $72^{\circ} \mathrm{C}$ for $10 \mathrm{~min}$ for elongation followed by $4^{\circ} \mathrm{C}$ for storage. Procedures for gene cloning, heterogeneous expression and protein purification were manipulated according to the manufacturer's manual. Plasmids constructed for this work are listed in Tab 3.

\section{Total RNA extraction, reverse transcription-PCR (RT-PCR)}

Total RNA was extracted from Leptospira strains cultured in EMJH medium for 24, 48 (log-phase), or144 h using Trizol Reagent (GBICO-BRL). The AMV Reverse Transcription System (Promega) was used for reverse transcription experiments. RT-PCR primers were designed to simultaneously detect the 3 ' portion of the vapB gene together with the 5' portion of the corresponding vapC gene (as shown in Tab 2). Reverse transcription synthesized first-strand cDNA was used as the PCR template. The amount of template cDNA used was 10-15 ng per assay. The PCR protocol was as described above, except for the annealing step which was done at $54^{\circ} \mathrm{C}$ for $1 \mathrm{~min}$.

\section{Bacterial growth curve detection}

Growth curves of the $E$. coli strains BL21(DE3/pLysS) containing pTB, pTC, pTBC were determined by measuring the O.D $D_{600 \mathrm{~nm}}$. Cells were initially grown in LB overnight with appropriate antibiotics (kanamycin, $50 \mu \mathrm{g} / \mathrm{ml}$ ) and then, transferred into fresh LB at $1 \%$ inoculums in the presence of IPTG $(1 \mathrm{mM})$.

\section{Plasmid stability test}

Single colonies of $E$. coli $\mathrm{DH} 5 \alpha$ carrying plasmid pUC19, pUB, pUC, or pUBC were picked from LB plates containing ampicillin $(50 \mu \mathrm{g} / \mathrm{ml})$ and innoculated into $5 \mathrm{ml}$ of LB without ampicillin. After shaking at $37^{\circ} \mathrm{C}, 220 \mathrm{rpm}$ for $12 \mathrm{~h}$, cells were reinnoculated into $5 \mathrm{ml}$ of fresh LB without ampicillin, but containing $1 \mathrm{mM}$ IPTG (1\% innoculum) for a further $12 \mathrm{~h}$. This kind of reinnoculation and cultivation was continued for up to $336 \mathrm{~h}$. $100 \mu \mathrm{l}$ samples were collected every $24 \mathrm{~h}$, diluted and plated onto LB-plates, with or without ampicillin $(50 \mu \mathrm{g} / \mathrm{ml})$. Viable colonies were counted after $12 \mathrm{~h}$ incubation at $37^{\circ} \mathrm{C}$. The ratio of the $A \mathrm{mp}^{\mathrm{R}}$ colonies versus the total viable colony counts was used to estimate the percentage of plasmid maintained in the population.

\section{RESULTS}

\section{The expression level of $\operatorname{vap} C$ in $E$. coli without or with the co-expression of $v a p B$ differed significantly}

Among the 4,769 predicted genes of L. interrogans (based on its genomic sequence - http://www.chgc.sh. $\mathrm{cn} / \mathrm{lep} /$ ), a pair of closely linked CDS (LA1002/LA1001) was shown to be strikingly similar to the bacterial virulence association proteins (VapB/VapC) [23], as well as a plasmid maintenance stability determinant locus, $(s t b)$ from Shigella flexneri[19].

The leptospira vapBC locus consists of an upstream $231 \mathrm{bp} \operatorname{CDS}(v a p B)$ that encodes a putative protein of 77 amino acids and a downstream 399 bp CDS (vapC) that encodes a putative protein of 133 amino acids (Fig 1). The translational start codon for vapC overlaps with the last base of the translational stop codon of vapB, which is a strong indication of translational coupling. This vapBC operon structure is strikingly similar to that of the three known TA operons, $\operatorname{relBE}[1,24], \operatorname{maz} E F[1]$ and $\operatorname{ch} p[3,25]$. A putative promoter region was revealed by the presence 


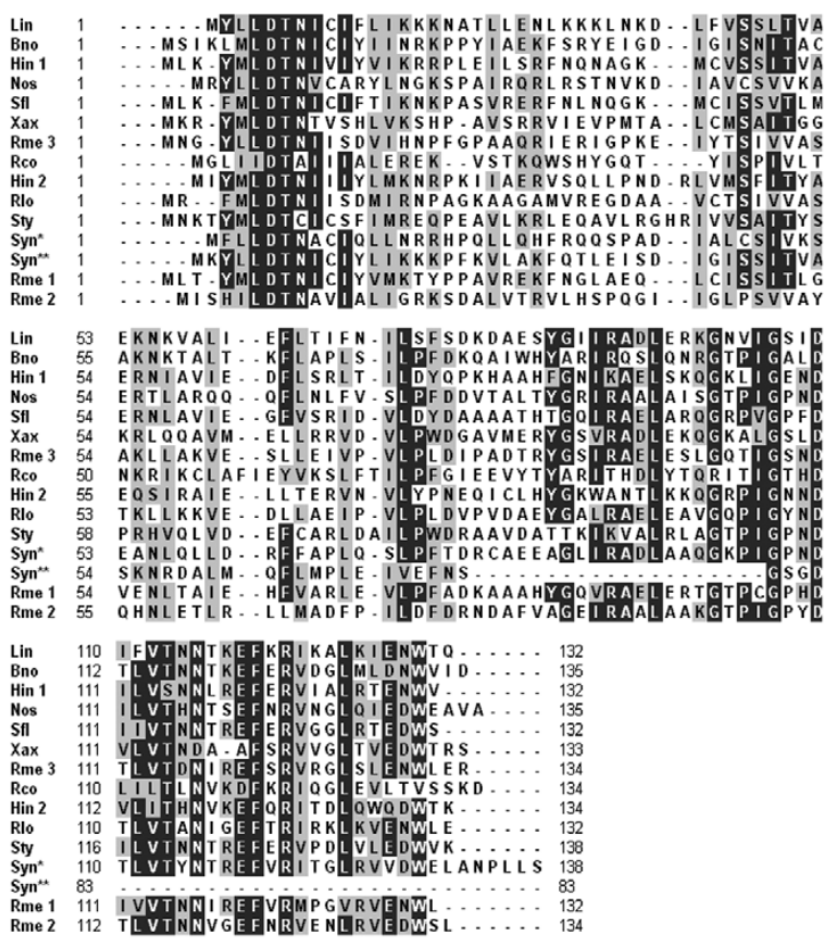

Fig 1. Multiple-sequence alignment of 18 VapC-homolog proteins from $L$. interrogans and other bacteria with similarity scores above e-8. Only the VapC proteins with obvious upstream VapB partners were included in the alignment analysis. Identical amino acids are shown in black, while conserved amino acids are shown in grey. The alignment was accomplished using Bioedit software. Gaps introduced to maximize the similarity alignment are indicated by dashes. The bacterial abbreviations used are listed in Tab 4.

of a pair of 9 bp inverted repeats (IR). This shares the same characteristics with IRs of the E. coli chp promoter, known for specific DNA binding of the Chp/Pem protein [26]. Both the -10 and -35 regions for the promoter and ribosomal binding site (RBS) are typical for bacteria (Fig 2).

In order to characterize this pair of predicted proteins, we cloned the $v a p B$ and $v a p C$ genes into $\mathrm{pET}$ expression vectors, either individually or together (Tab 3) and attempted to express the genes in E. coli BL21(DE3 pLysS) (Novagen). The recombinant proteins were expressed after IPTG induction and shown to have a molecular weight (MW) of $10.7 \mathrm{kD}$ for VapB with his-tag and $17.1 \mathrm{kD}$ for VapC with his-tag (Fig 3). The expression level of VapC was significantly lower than that of its partner VapB if expressed alone. However, when these two proteins were expressed simultaneously and coordinately from a construct containing their original operon with the characteristic coupled cistronic structure (Fig 2), the expression levels of both were high and essentially the same (Fig 3).

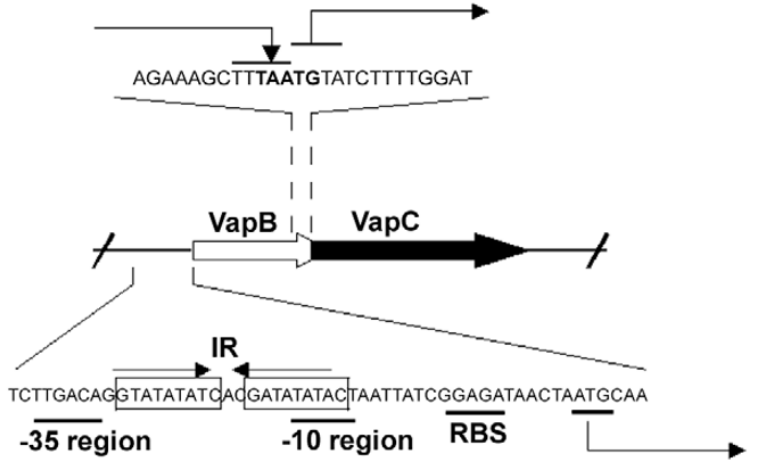

Fig 2. Schematic representation of the putative promoter region of the chromosome-encoded $v a p B C$ of L. interrogans. Single arrows show all of the translation directions. The overlap of the stop codon of $v a p B$ with the start codon of $v a p C$ is enlarged. The 9-bp inverted repeats (IRs) are boxed and highlighted by two head-tohead arrows. Typical -10 and -35 regions as well as the ribosomebinding site (RBS) are marked and the vapB start coden is bolded.

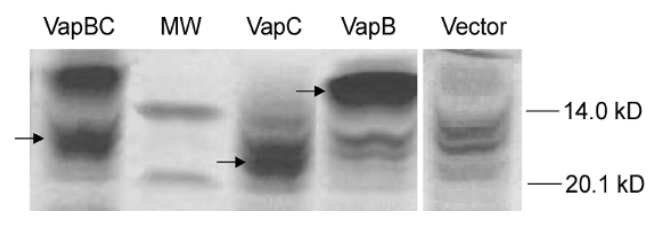

Fig 3. Proteins encoded by vapB and $\operatorname{vap} C$ clones expressed in $E$. coli BL21 (DE3/pLysS). The proteins were expressed as described in Materials and Methods. The arrows indicate each expressed Histag recombinant protein.

\section{The growth of the $E$. coli strain carrying the vapC expression plasmid was restrained but was relieved with the co-expression of the vapB gene}

The growth of the E. coli carrying plasmid pTC ( $\operatorname{vapC}$ expression plasmid) sharply decreased in the presence of IPTG compared to that of the hosts with plasmids pTB (vapB expression plasmid), pTBC (vapBC coupled expression plasmid) or $\mathrm{pET} 28 \mathrm{~b}$ (the negative control vector) (Fig 4). Quantitatively, there was no difference in their doubling times ( $44 \mathrm{~min}$ for pTC, $47 \mathrm{~min}$ for $\mathrm{pTB}, 45 \mathrm{~min}$ for pTBC and $46 \mathrm{~min}$ for pET28b). However, the difference was significant for the length of the lag phase (248 min for $\mathrm{pTC}$ but 28 min for $\mathrm{pTB}, 40$ min for $\mathrm{pTBC}$, and $34 \mathrm{~min}$ for $\mathrm{pET} 28 \mathrm{~b}$ ). This difference directly leads to the difference in the speed of accumulation of biomass. At the $14^{\text {th }} \mathrm{h}$ of cultivation, the average $\mathrm{OD}_{600}$ reading of $E$. coli harboring pTC (1.15) was much lower than that of all the other 3 strains (1.43 for pTB, 1.49 for $\mathrm{pTBC}$ and 1.51 for pET28b). 


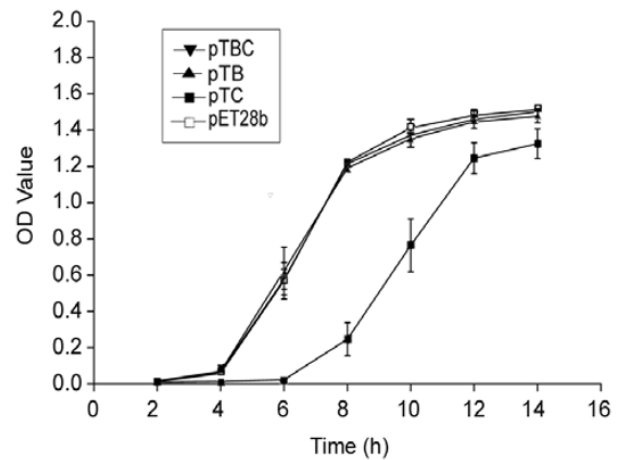

Fig 4. Effect of the over-expression of the $L$. interrogans vapC in the presence or absence of $v a p B$ on growth of $E$. coli cells harboring the expression plasmids. Symbols used for the plasmids transformed to the $E$. coli hosts: pET28b $(\square)$, pTB $(\boldsymbol{\Delta})$, pTC $(\boldsymbol{\square})$, pTBC $(\boldsymbol{\nabla})$. Growth was determined by measuring the optical density at $600 \mathrm{~nm}$.

A

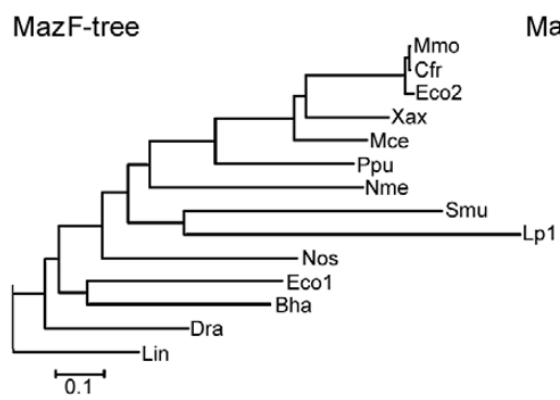

MazE-tree (Lactobacillus plantarum has no mazE)

MazEF-16S-tree
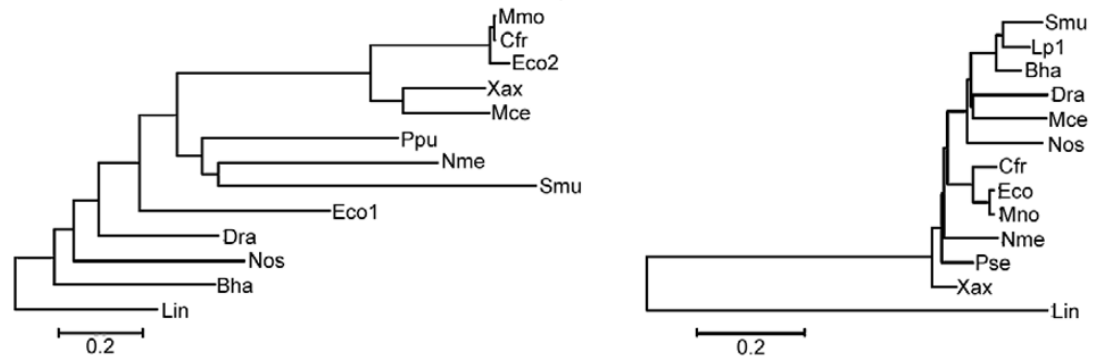

B
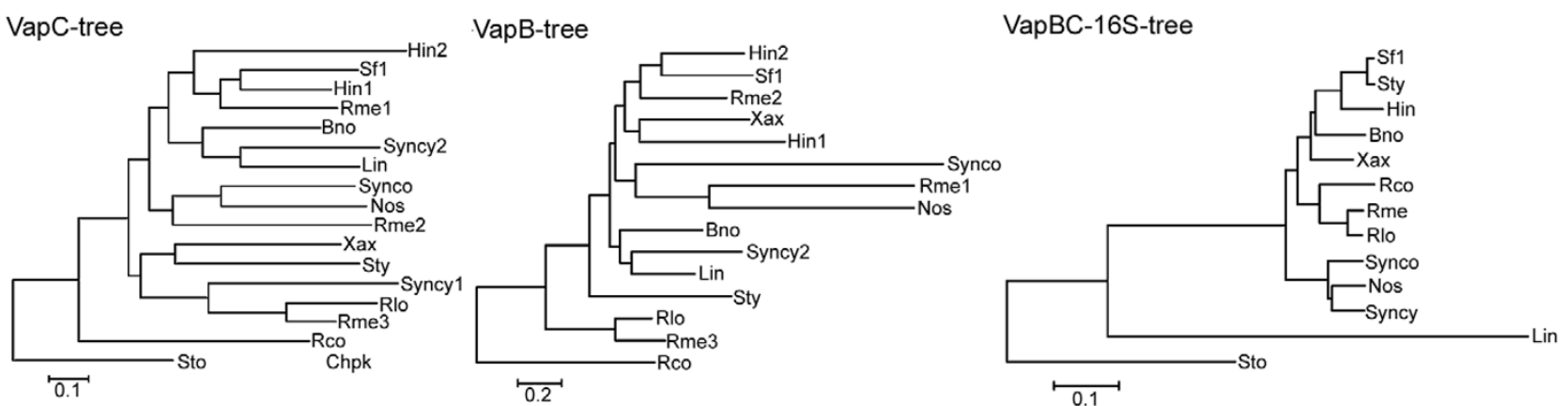

C

Chpk-tree

Chpl-tree
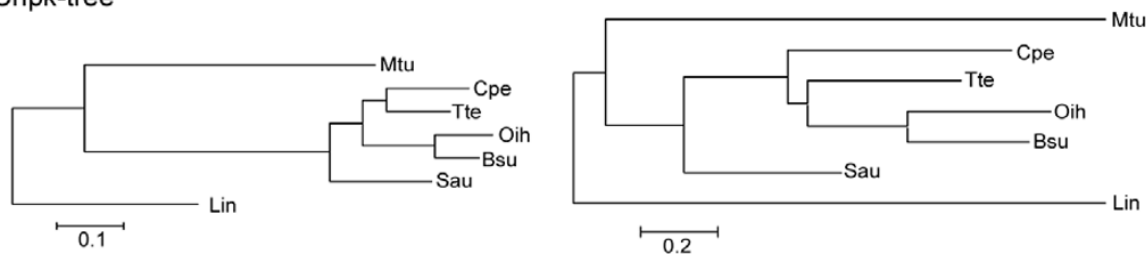

ChplK-16S-tree

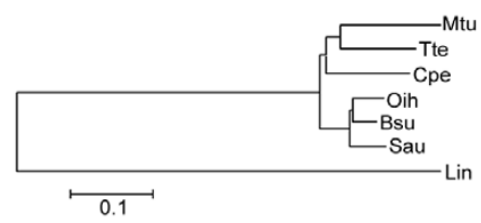

Fig 5. Unrooted phylogenetic trees for the amino acid sequences of bacterial antitoxin-toxin MazEF (panel A), VapBC (panel B) and ChpIK (panel C) and 16s rRNA sequences corresponding to each kind of TA system. In panel B, S. tokodaii was drawn as the root in the VapC tree because this archeae strain is the most primitive among all the bacteria in this group. As $S$. tokodaii does not have a vapB gene, $R$. conorii, the strain closet to $S$. tokodaii in the tree for VapC, was drawn as the root in the VapB tree. All the other trees were unrooted. The abbreviations for the bacterial species are listed in Tab 4. 
Tab 4. The chromosome-encoded TA systems distributed in microorganisms

\begin{tabular}{|c|c|c|c|c|c|c|c|c|c|}
\hline Abbreviation & Bacterial species/strains & $\operatorname{vap} B$ & $\operatorname{vapC}$ & $\operatorname{maz} E$ & $m a z F$ & $\mathrm{relB}$ & relE & $\operatorname{chpK}$ & chpI \\
\hline Lin & Leptospira interrogans & LA1002 & LA1001 & LA1780 & LA1781 & & & LA2843 & LA2844 \\
\hline \multirow[t]{2}{*}{ Eco } & Escherichia coli & & & P18534 & P33645 & P07007 & P07008 & & \\
\hline & & & & P13975 & P13976 & Q8VU39 & Q8VU40 & & \\
\hline Sfl & Shigella flexneri & O06663 & O06662 & & & & & & \\
\hline \multirow[t]{2}{*}{ Hin } & Haemophilus influenzae & Q57534 & P71363 & & & & & & \\
\hline & & Q57120 & Q57122 & & & & & & \\
\hline \multirow[t]{3}{*}{ Rme } & Rhizobium meliloti & Q92KY4 & Q92KY5 & & & NO & Q92Q75 & & \\
\hline & & Q92ME1 & Q92ME0 & & & & & & \\
\hline & & Q9L381 & Q9L380 & & & & & & \\
\hline Xax & Xanthomonas axonopodis & Q8PRH2 & Q8PRH3 & Q8PRN0 & Q8PRN1 & & & & \\
\hline \multirow[t]{2}{*}{ Sty } & Salmonella typhimurium & Q8L236 & Q8L237 & & & Q8ZPG0 & Q8ZPF9 & & \\
\hline & & & & & & Q8XES7 & Q8XGL8 & & \\
\hline Rlo & Rhizobium loti & Q98K21 & Q98K22 & & & & & & \\
\hline Rco & Rickettsia conorii & Q92IP0 & Q92IP1 & & & & & & \\
\hline Bno & Bacteroides nodosus & Q46558 & Q46557 & & & & & & \\
\hline Synco & Synechococcus sp. & Q9Z3G8 & Q9Z3G9 & & & & & & \\
\hline Nos & Nostoc sp. & Q8YK90 & Q8YK91 & Q8YS79 & Q8YS80 & & & & \\
\hline \multirow[t]{2}{*}{ Syncy } & Synechocystis sp. & NO & Q55189 & & & & & & \\
\hline & & P72994 & P72993 & & & & & & \\
\hline $\mathrm{Bsu}$ & Bacillus subtilis & & & & & & & P96622 & P96621 \\
\hline Dra & Deinococcus radiodurans & & & Q9RX99 & Q9RX98 & & & & \\
\hline Nme & Neisseria meningitides & & & Q9JZS7 & Q9JZS8 & & & & \\
\hline $\mathrm{Ppu}$ & Pseudomonas putida & & & AAN66395 & AAN66396 & & & & \\
\hline \multirow[t]{2}{*}{$\mathrm{Smu}$} & Streptococcus mutans & & & Q8DW96 & Q8DW95 & & & & \\
\hline & UA159 & & & & & & & & \\
\hline Mce & Mycobacterium celatum & & & Q93S65 & Q93S64 & & & & \\
\hline Mtu & Mycobacterium tuberculosis & & & & & & & Q10867 & Q10868 \\
\hline $\mathrm{Cfr}$ & Citrobacter freundi & & & Q8GFY0 & Q8GFY1 & & & & \\
\hline Mmo & Morganella morganii & & & O52204 & O52205 & Q9ZH43 & Q9ZH42 & & \\
\hline Lpl & Lactobacillus plantarum & & & NO & gp|AL935260 & & & & \\
\hline Oih & Oceanobacillus iheyensis & & & & & & & BAC12579 & Q8ESW4 \\
\hline \multirow[t]{2}{*}{ Tte } & Thermoanaerobacter & & & & & & & Q8R861 & Q8R5Q2 \\
\hline & tengcongens & & & & & & & & \\
\hline Cpe & Clostridium perfringens & & & & & & & Q8XNN7 & Q8XNN8 \\
\hline Sau & Staphylococcus aureus & & & & & & & O05341 & Q99SI6 \\
\hline Asa & Aeromonas salmonicida & & & & & CAD48430 & Q8GMN9 & & \\
\hline Vch & Vibrio cholerae & & & & & Q08800 & Q08799 & & \\
\hline Bha & Bacillus halodurans & & & Q9K6K9 & Q9K6K8 & & & & \\
\hline Sto & Solfolobus tokodaii & NO & Q96Z88 & & & & & & \\
\hline
\end{tabular}

The protein sequences codes are from Swiss-Prot or TrEMBL. 


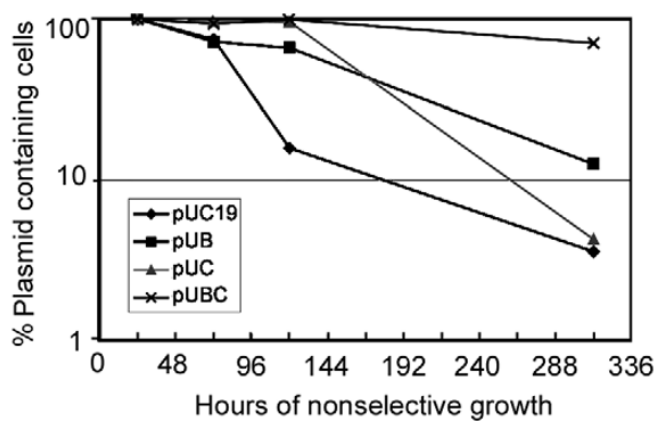

Fig 6. Genetic stabilization effect of the $\operatorname{vap} B C$ system upon the high-copy unstable plasmid, pUC19. Plasmids used were derivatives of pUC19: pUB (carrying vapB), pUC (carrying vapC), pUBC (carrying vapBC) and pUC19 (blank control). Plasmid construction and experimental details are described in Materials and Methods.

\section{The vapBC locus is conserved in different species or serovars of Leptospira}

The presence of the vapBC locus and the expression of this operon was tested in various kinds of pathogenic or saprophytic leptospires (Tab 1). PCR amplification of vapBC genes from genomic DNA templates of the experimental strains showed that both the 231 bp vapB fragment and the $399 \mathrm{bp}$ vapC were present in all the strains tested (data not shown). Subsequent DNA sequencing results showed that the strains tested had identical gene fragments in $\operatorname{vapB}$ and $\operatorname{vap} C$ (data not shown). RT-PCR experiments employing total RNA isolated from cells grown on EMJH medium with gene specific primers (see Materials and Methods) showed that these two closely linked genes were transcribed simultaneously in all the Leptospira strains tested (data not shown).

\section{The $L$. interrogans vapBC system is effective in stabilizing pUC plasmid in $E$. coli}

The stability of a series of $\mathrm{pUC} 19$ derived plasmids containing vapB (pUB), vapC (pUC), or vapBC (pUBC) genes with the cloned genes expressed by the lac promotor of pUC19 were measured in E. coli DH5 $\alpha$ (see Materials and Methods). As shown in Fig 6, the percentage of ampicilin resistant cells in the population of $E$. coli carrying pUC19 decreased. On the other hand, the majority of $E$. coli cells carrying pUBC with $\operatorname{vap} B C$ were relatively stable after prelonged incubation without any selection pressure. Neither pUC nor pUB was stable under the same conditions, although the rate of plasmid lost was slightly improved.

\section{DISCUSSION}

The vap module was first found in the genome of the strictly anaerobic bacterium, Dichelobacter nodosus (Bacteroides nodosus)[18], a major pathogen implicated in foot rot (a highly contagious disease of sheep). Hybridization experiments showed that the vap module was present in all the genomes of virulent isolates, while it was absent in $67 \%$ of the benign isolates. Therefore, it was named the virulent-associated region and the hypothetical proteins encoded in this region were designated as virulent-associated proteins (vap), although their definitive functions were unknown.

Subsequently, many vapBC loci were found in other bacteria (Fig 1, Tab 4), encoded by either chromosomes or plasmids and in particular, the $s t b$ locus encoded on the large virulent plasmid pMYSH6000 of S. flexneri, are both structurally and functionally similar to the vap system. By studying the physiology of the overexpression of $L$. interrogans vap $B C$ genes in $E$. coli, we recognized that, similar to the case of L. interrogans chpIK operon[3], $v a p B C$ is a novel type of toxin-antitoxin system. In contrast to $B$. nodosus, the vapBC operon is widely present and expressed in leptospires. Therefore, it is more likely related to physiological regulation rather than to the virulence of the bacterium.

Deduced amino acid sequence alignment of the VapC proteins indicated that besides the general similarity among the family members, highly conserved specific sites were observed (Fig 1). All the VapC proteins contain a common PIN domain (IPR002716, InterPro) putatively responsible for nucleic acid binding, which might infer the possibility of a common cellular target for the Vap TA system.

Further similarity searches and comparisons in the microbial genomic database with respect to TA systems revealed a total of four types encoded by bacterial chromosomes. They are the relBE, mazEF, chpIK, and vapBC loci (Tab 4). Except for the chpIK locus, all the others are also found in plasmids (Tab 4).

It is interesting to note that several TA systems, or multiple copies of one TA system, may present in one genome (Tab 4). In this connection, L. interrogans stands out as the only bacterium that possesses three TA systems, the chpIK previously identified[3], the $v a p B C$ characterized in this study, and the mazEF (LA1780/LA1781) recognized by homology searches. It is known[21] that TA systems encoded by plasmids prevent plasmid loss at cell division, whereas TA loci found on chromosomes are associated with species stability or cell programmed death (bacterial apoptosis). Further studies have revealed the specific molecular targets of some of the toxins, i.e., CcdB inhibits DNA gyrase, while PemK/Kid inhibits DNA replication, presumably via interaction with the DnaB helicase[27]. The RelE proteins encoded by the $E$. coli K12 chromosome presumably inhibit translation[2, 20], but 
their specific target(s) within the translational machinery is not yet known. Recent studies have shown that the ChpK proteins of E.coli inhibit cell growth by cleavage of mRNA [28]. Similarly, RelE toxin inhibits protein synthesis by cleavage of ribosome bound mRNA in response to nutritional stress[29]. These findings further support the hypothesis proposed by Gerdes[21]. Toxins from the TA gene families, the RelE, VapC, MazF and ChpK, may mediate inhibition of translation or DNA replication[21] as part of the global cellular response to environmental stress, rather than being simply cell-killing. The presence of multiple TA systems in one particular genome, as shown in the case of L. interrogans, may imply the complexity of regulation during the transition of metabolism.

Co-evolution is usually represented by a change in genetic composition of one gene in response to the genetic change in another[30]. The translational coupling of two closely linked genes with a strongly interactive functional relationship to the proteins they encoded, such as the case of TA system, seems to enforce the strongest selection pressure for co-evolution. Amino acid sequence similarity and clustering analysis of each group of the TA system were carried out and the results were compared to their corresponding 16s RNA phylogenetic trees (Fig 5). In general, the co-evolution between toxins and their corresponding antitoxins is obvious, while the development of these TA systems along with their speciation route varies from case to case. It is also interesting to note that homologous analysis could spontaneously put the toxin sequences into four groups, but not for their antitoxins (data not shown). These observations indicate that quite a few of the TA systems did not originally exist in the species, i.e., horizontal gene transfer might account for significant portions of the event. Therefore, co-evolution of the toxin genes, as well as the antitoxin genes with the 16s RNA, is only observed in part of the species as individual groups, but not along the whole family. This phenomenon is clearly illustrated in the case of the MazEF family (Fig 5A) where most of the Gram-negative bacteria except Nostoc, are grouped together as that of their speciation. However, Gram-positive bacteria are grouped separately into different Gram-negative bacterial clusters. Furthermore, even for each TA system, the toxin and antitoxin may not have originated from the same ancestor, or even been acquired simultaneously. This phenomenon is particularly obvious in case where multiple copies of one TA system existed in one strain. For instance, Rhizobium meliloti has two sets of VapBC systems encoded by its genome, but the phylogenetic position VapB1 only matches with that of VapC2 and vice versa. This implies not only an independent origination, but also a possible trans-acting mechanism between two TA modules of the same category (Fig 5B). In addition, it seems that if the evolving of a TA system among bacteria was a relatively homogeneous process, e.g., without gene duplication so that only a single copy of a TA module existed in one species (as is the case of chpIK) parallel evolution between the TA module and the 16s RNA could be observed (Fig 5C). A similar situation was observed in the family of RelBE, which consists of Gramnegative bacteria only (data not shown). This fact implies that the evolvement of relBE module might be late in the evolution history and it might have no chance to be spread into bacteria other than the Gram-negative ones. Finally, we noticed that in certain cases, even the strong co-evolutionary relationship between toxin and antitoxin was distorted. For instance, antitoxin was not found in the only archeae that possessed a toxin gene (vapC), Solfolobus tokodaii. This is interesting because it suggests that the function of the toxin-like proteins found in archeae might not necessarily be the same as originally thought. Alternatively, it is equally possible that although these proteins are toxic to the host, they might not be functionally expressed under the physiological conditions of these hosts. In either case, it suggests that antitoxins are likely introduced into the system later in the evolutionary path, and that the genetic signature of this "double evolving" event is still embedded in the coding sequence that is seen today.

\section{CONCLUSIONS}

Our data have demonstrated that the $\operatorname{vap} B C$ locus of $L$. interrogans belongs to the TA family rather than encoding virulence-associated proteins. The product of the vapC gene is toxic to $E$. coli cells, while the product of the $v a p B$ gene counteracts VapC toxicity. The presence of the vapBC operon on an unstable plasmid under the control of a $l a c$ promoter can effectively prevent plasmid loss under nonselective conditions. A pair of $9 \mathrm{bp}$ IRs in the promoter region of the L. interrogans vapBC operon share the same characterization with IRs of the $E$. coli $\operatorname{chp} B$ promoter region, indicating it might be a specific DNA binding site for VapC proteins. At present, the cellular target of VapC protein remains unknown. Further study on $L$. interrogans VapB and VapC could help in the understanding of the cellular target of these toxins and their physiological role in bacteria.

\section{ACKNOWLEDGEMENTS}

We thank Xiu Gao JIANG (The Institute of Epidemiology and Microbiology, Chinese Academy of Preventive Medicine) and Bao Yu HU (Department of Microbiology and Parasitology, Shanghai Second Medical University) for kindly providing Leptospira strains and cultures. We also thank You Gang MIAO (Shanghai Information Center for Life Sciences, Chinese Academy of Sciences), Hai XU 
(Institute of Plant Physiology \& Ecology, Chinese Academy of Sciences), Hui Feng XI and Lu Ling FENG (CHGCS) for their support in the bioinformatics analysis. This work was supported by the National High Technology Research and Development Program of China (Program No. 2003AA223031).

Received, Oct 21, 2003

Revised, Apr 25, 2004

Accepted, Apr 28, 2004

\section{REFERENCES}

1 Aizenman E, Engelberg-Kulka H, Glaser G. An Escherichia coli chromosomal "addiction module" regulated by guanosine 3', 5'bispyrophosphate: a model for programmed bacterial cell death. Proc Natl Acad Sci 1996; 93:6059-63.

2 Gotfredsen M, Gerdes K. The Escherichia coli relBE genes belong to a new toxin-antitoxin gene family. Mol Microbiol 1998; 29:1065-76.

3 Picardeau M, Ren S, Saint Girons I. Killing effect and antitoxic activity of the Leptospira interrogans toxin-antitoxin system in Escherichia coli. J Bacteriol 2001; 183:6494-7.

4 Faine S, Adler B, Bolin C, Perolat P. Leptospira and Leptospirosis. 1999; Medisci Melbourne.

5 Ren SX, Fu G, Jiang XG, et al. Unique physiological and pathogenic features of Leptospira interrogans revealed by whole-genome sequencing. Nature 2003; 422:888-93.

6 Fraser CM, Norris SJ, Weinstock GM et al. Complete genome sequence of Treponema pallidum, the syphilis spirochete. Science 1998; 281:375-88.

7 Fraser CM, Casjens S, Huang WM et al. Genomic sequence of a Lyme disease spirochaete, Borrelia burgdorferi. Nature 1997; 390:580-6.

8 Gerdes K. Toxin-antitoxin modules may regulate synthesis of macromolecules during nutritional stress. J Bacteriol 2000; 182: $561-72$.

9 Holcik M. Lyer VN. Conditionally lethal genes associated with bacterial plasmids. Microbiology 1997; 143:3403-16.

10 Jensen RB, Gerdes K. Programmed cell death in bacteria: proteic killer gene systems. Mol Microbiol 1995; 17:205-10.

11 Kobayashi I. Selfishness and death: raison d'etre of restriction, recombination and mitochondria. Trends Genet 1998; 14:36874.

12 Bahassi EM, O’Dea MH, Allali N, Messens J, Gellert M, Couturier M. Interactions of CcdB with DNA gyrase. Inactivation of GyrA, poisoning of the gyrase-DNA complex, and the antidote action of CcdA. J Biol Chem 1999; 274:10936-44.

13 Tam JE, Kline BC. The F plasmid $c c d$ autorepressor is a complex of CcdA and CcdB proteins. Mol Gen Genet 1989; 219:26-32.

14 Ruiz-Echevarria MJ, Torre MA, Diaz-Orejas RA mutation that decreases the efficiency of plasmid $\mathrm{R} 1$ replication leads to the activation of parD, a killer stability system of the plasmid. FEMS Microbiol Lett 1995; 130:129-5.

15 Gazit E, Sauer RT. The Doc toxin and Phd antidote proteins of the bacteriophage P1 plasmid addiction system form a heterotrimeric complex. J Biol Chem 1999; 274:16813-8.

16 Johnson EP, Ström AR, Helinski DR. Plasmid RK2 toxin protein ParE: purification and interaction with the ParD antitoxin protein. J Bacteriol 1996; 178:1420-9.

17 Smith AS, Rawlings DE. The poison-antidote stability system of the broad-host-range Thiobacillus ferrooxidans plasmid pTFFC2. Mol Microbiol 1997; 26:961-970.

18 Ceglowski P, Boitsov A, Chai S, Alonso JC. Analysis of the stabilization system of pSM19035-derived plasmid pBT233 in Bacillus subtilis. Gene 1993; 136:1-12.

19 Radnedge L, Davis MA, Youngren B, Austin SJ. Plasmid maintenance functions of the large virulence plasmid of Shigella flexneri. J Bacteriol 1997; 179:3670-5.

20 Grønlund H, Gerdes K. Toxin-antitoxin systems homologous to relBE of Escherichia coli plasmid P307 are ubiquitous in prokaryotes. J Mol Biol 1999; 285:1401-15.

21 Kenn Gerdes. Toxin-Antitoxin Modules May Regulate Synthesis of Macromolecules during Nutritional Stress. J Bacteriol 2000; 182:561-72.

22 Segers RP, van der Drift A, de Nijs A, Corcione P et al. Molecular analysis of a sphingomyelinase C gene from Leptospira interrogans serovar hardjo. Infect Immun 1990; 58:2177-85.

23 Katz ME, Howarth PM, Yong WK et al. Identification of three gene regions associated with virulence in Dichelobacter nodosus, the causative agent of ovine footrot. J Gen Microbiol 1991; 137:2117-24.

24 Gotfredsen M, Gerdes K. The Escherichia coli relBE genes belong to a new toxin-antitoxin gene family. Mol Microbiol 1998; 29:1065-76.

25 Masuda Y, Miyakawa K, Nishimura Y et al. $\operatorname{chp} A$ and $\operatorname{chp} B$, Escherichia coli chromosomal homologs of the pem locus responsible for stable maintenance of plasmid R100. J Bacteriol 1993; 175:6850-6.

26 Bravo A, de Torrontegui G, Diaz R. Identification of components of a new stability system of plasmid R1, ParD, that is close to the origin of replication of this plasmid. Mol Gen Genet 1987; 210:101-10.

27 Ruiz-Echevarria MJ, Gimenez-Gallego G, Sabariegos-Jareno R, Diaz-Orejas R. Kid, a small protein of the parD stability system of plasmid R1, is an inhibitor of DNA replication acting at the initiation of DNA synthesis. J Mol Biol 1995; 247:568-77.

28 Christensen SK, Pedersen K, Hansen FG et al. Toxin-antitoxin loci as stress-response-elements: ChpAK/MazF and ChpBK cleave translated RNAs and are counteracted by tmRNA. J Mol Biol 2003; 332:809-19.

29 Pedersen K, Zavialov AV, Pavlov MY et al. The bacterial toxin RelE displays codon-specific cleavage of mRNAs in the ribosomal A site. Cell 2003; 112:131-40.

30 Pal C, Hurst LD. Evidence for co-evolution of gene order and recombination rate. Nat Genet 2003; 33:392-5. 